\title{
Erratum: Narrow dip inside a natural linewidth absorption profile in a system of two atoms [Phys. Rev. A 92, 053840 (2015)]
}

\author{
A. A. Makarov \\ (Received 26 September 2016; published 10 October 2016)
}

DOI: 10.1103/PhysRevA.94.049901

In this paper, Eq. (39) should be corrected to

$$
\gamma \approx \frac{3(k r)^{2} \Gamma}{32 \pi} \int_{0}^{2 \pi} \int_{0}^{\pi}(\sin \alpha \sin \theta \cos \varphi+\cos \alpha \cos \theta)^{2} \sin ^{3} \theta d \theta d \varphi=\frac{1}{8}(k r)^{2} \Gamma\left[\frac{2}{3}+\frac{1}{5}\left(2 \cos ^{2} \alpha-\sin ^{2} \alpha\right)\right] .
$$

Also, Eq. (45) should be replaced with

$$
\begin{aligned}
& \left\|U_{\mathrm{s}}\right\|=U_{r}\left\|\begin{array}{ccc}
1-\frac{3}{2} \sin ^{2} \alpha & -\frac{3 \sqrt{2}}{4} \sin 2 \alpha & \frac{3}{2} \sin ^{2} \alpha \\
-\frac{3 \sqrt{2}}{4} \sin 2 \alpha & 1-3 \cos ^{2} \alpha & \frac{3 \sqrt{2}}{4} \sin 2 \alpha \\
\frac{3}{2} \sin ^{2} \alpha & \frac{3 \sqrt{2}}{4} \sin 2 \alpha & 1-\frac{3}{2} \sin ^{2} \alpha
\end{array}\right\|, \\
& \mid U_{\mathrm{a}}\left\|=U_{r}\right\| \begin{array}{ccc}
\frac{3}{2} \sin ^{2} \alpha-1 & \frac{3 \sqrt{2}}{4} \sin 2 \alpha & -\frac{3}{2} \sin ^{2} \alpha \\
\frac{3 \sqrt{2}}{4} \sin 2 \alpha & 3 \cos ^{2} \alpha-1 & -\frac{3 \sqrt{2}}{4} \sin 2 \alpha \\
-\frac{3}{2} \sin ^{2} \alpha & -\frac{3 \sqrt{2}}{4} \sin 2 \alpha & \frac{3}{2} \sin ^{2} \alpha-1
\end{array} \| .
\end{aligned}
$$

The corrected forms of Eqs. (39) and (45) were actually used in our calculations. Therefore, these corrections do not affect any other results of the paper. 